\title{
Lo Transindividual: de Simondon a Marx ${ }^{1}$
}

\author{
Daniel Alvaro $^{2}$
}

\begin{abstract}
RESUMEN: El presente artículo trata de lo transindividual, un concepto fundamental en la teoría de la individuación elaborada por Gilbert Simondon (1924-1989), y de su vinculación con ciertas proposiciones de Karl Marx que tratan del individuo y la sociedad. Entre ambos autores existe una relación directa, evidenciada en cada uno de los textos donde Simondon hace mención a Marx y al marxismo en general. Pero también existe una relación indirecta, para nada evidente, que surge menos de lo que Simondon dice acerca de Marx que de lo que ciertos pensadores contemporáneos dicen sobre uno y otro. Aquí intento dar cuenta de ambos tipos de relaciones. Asimismo, me propongo introducir y comentar algunos pasajes de Marx que compiten en radicalidad y audacia con los que escribiera Simondon un siglo más tarde, y en los cuales quiero apoyar mi hipótesis de lectura, a saber, que Marx es un pensador de lo transindividual avant la lettre.
\end{abstract}

PALABRAS CLAVE: Transindividual. Simondon. Marx. Relación.

De un tiempo a esta parte Gilbert Simondon (1924-1989) dejó de ser un autor para unos pocos iniciados. Su obra, concebida en Francia en las décadas del '50 y del '60, tuvo una temprana aceptación pero el amplio reconocimiento de la misma es relativamente reciente. Desde hace al menos dos décadas sus aportes en el campo de la filosofía de la técnica, la ontología, la estética, la psicología y las ciencias sociales comenzaron a ser revalorizados dentro y fuera de Francia. Hoy sus libros se encuentran disponibles en ediciones nuevas y mejoradas, y buena parte de su producción inédita (compuesta principalmente por cursos, conferencias, artículos y entrevistas) está siendo publicada y traducida a diversas lenguas. Asimismo, se celebran coloquios

\footnotetext{
${ }^{1}$ http://dx.doi.org/10.1590/S0101-31732016000400008

${ }^{2}$ Es investigador del Consejo Nacional de Investigaciones Científicas y Técnicas (CONICET). E-mail: danielalvaro@gmail.com
}

Doctor en Ciencias Sociales por la Universidad de Buenos Aires (UBA) y Doctor en Filosofía por la Université Paris 8 Vincennes - Saint-Denis. Es investigador del Consejo Nacional de Investigaciones Científicas y Técnicas (CONICET) con sede en el Instituto de Investigaciones Gino Germani de la UBA, y es docente de la materia "Teoría Estética y Teoría Política", perteneciente a la carrera de Sociología de la misma universidad. Desarrolla actividades docentes y dicta seminarios de posgrado en distintas universidades latinoamericanas. Publica artículos y ensayos y ha traducido obras de Jacques Derrida y Jean-Luc Nancy. Es coordinador y coautor de Jean-Luc Nancy: arte, filosofía, política (Buenos Aires: Prometeo, 2012) y autor de El problema de la comunidad. Marx, Tönnies, Weber (Buenos Aires: Prometeo, 2015). E-mail: danielalvaro@gmail.com 
y homenajes dedicados a su obra en todo el mundo. Se puede afirmar con seguridad que Simondon ya no pasa ni pasará desapercibido.

Una de las razones más importantes para explicar este fenómeno es su arrolladora actualidad o, si se prefiere, su contemporaneidad. Tanto más sugerente cuanto que resulta intempestiva. En cada una de las disciplinas en donde incursionó, su perspectiva viene a transformar y en ciertos casos a revolucionar los paradigmas existentes, desde los más antiguos hasta los más recientes, incluidos algunos que lo sucedieron en el tiempo. Simondon se movía con comodidad entre las disciplinas como pocos pensadores de su tiempo, y lo hacía de modo tal que cualquier distinción taxativa entre teoría y práctica le resultaba inimaginable. Este doble gesto, más propio de nuestra época que de la suya, forma parte de su vigencia y hace de Simondon el autor indispensable en el que se ha convertido hoy en día.

El presente artículo escapa a cualquier pretensión de síntesis generalizadora de su pensamiento. ${ }^{3}$ Por el contrario, el problema que aborda es más bien específico. Se trata de la cuestión de lo transindividual, un concepto fundamental en la teoría de la individuación elaborada por Simondon, y de su vinculación con ciertas proposiciones de Marx que tratan del individuo y la sociedad. Como se verá, existe entre ambos una relación directa, evidenciada en cada uno de los textos donde Simondon hace mención a Marx y al marxismo en general. Pero también existe una relación indirecta, para nada evidente, que surge menos de lo que Simondon dice acerca de Marx que de lo que ciertos pensadores contemporáneos dicen sobre uno y otro.

En primer lugar, aquí se intenta dar cuenta de ambos tipos de relaciones. Por un lado, comentaré brevemente las críticas que Simondon le dirige a Marx en sus dos obras más importantes y, por lo demás, las únicas publicadas en vida del autor: La individuación a la luz de las nociones de forma $y$ de información y El modo de existencia de los objetos técnicos. ${ }^{4}$ Por otro lado, voy a referirme a textos recientes de Étienne Balibar y Paolo Virno donde se ofrecen pistas a seguir para indagar otra relación posible entre Simondon y

\footnotetext{
${ }^{3}$ Para una visión de conjunto sobre los problemas centrales de su teoría en los distintos campos disciplinares de los que participa, véase DE BOEVER et al., 2012.

${ }^{4}$ La primera de ellas corresponde a su tesis de doctorado principal y la segunda a su tesis de doctorado complementaria. Ambas fueron defendidas en 1958. Para información detallada sobre la publicación y recepción de las mismas puede consultarse el sitio creado por Nathalie Simondon: http://gilbert. simondon.fr.
} 
Marx, una relación que a diferencia de la anterior muestra más puntos de contacto que de separación entre uno y otro.

En segundo lugar, se trata de pensar la experiencia de la transindividualidad. ¿Qué es lo transindividual? ¿Qué implica desde un punto de vista lógico la afirmación de una realidad transindividual que no es ni simplemente psíquica ni simplemente colectiva, ni interior ni exterior, sino más bien la unidad de todos estos niveles de realidad? ¿Cómo se relaciona la apuesta de Simondon por la transindividualidad con su proyecto de fundar una nueva epistemología para pensar la individuación y los fenómenos psicosociales ligados a ella?

En tercer y último lugar, me propongo introducir y comentar algunos pasajes de Marx pertenecientes a distintas épocas donde la clásica oposición entre individuo y sociedad aparece seriamente cuestionada. Pasajes que compiten en radicalidad y audacia con los que escribiera Simondon un siglo más tarde, y en los cuales quiero apoyar mi hipótesis de lectura, a saber, que Marx es un pensador de lo transindividual avant la lettre.

\section{Trabajo, alienación y Más allá}

Como adelanté, los comentarios que Simondon le dedica a Marx en sus dos libros capitales tienen un alcance crítico. No obstante, una lectura detallada de los mismos mostraría que, por severos que puedan llegar a ser, no carecen de reconocimiento por la herencia teórica de Marx. Si bien las objeciones desplegadas en ambos libros no son las mismas, tienen, por decirlo así, un trasfondo común. En términos generales, Simondon le critica a "Marx" y al "marxismo" — sin distinguir verdaderamente entre uno y otro — un cierto esquematismo y una limitación en el análisis de la relación entre los hombres y la naturaleza y, más en concreto, en el análisis del trabajo y de la alienación. Se trata de juicios agudos que apuntan a conceptos centrales de la teoría marxista. Al margen de la consistencia, incluso de la pertinencia de estas críticas, quisiera llamar la atención sobre el posicionamiento de Simondon, sobre la distancia que toma respecto de Marx y del marxismo. Esta cuestión me interesa básicamente por dos razones. En primer lugar, porque no es la única vez que Simondon plantea objeciones a la teoría de un autor de la que sin embargo se encuentra próximo o con la que incluso parece coincidir. También sucede con Spinoza, y en este caso, quizás, de manera aún más incontestable dada la convergencia entre la comprensión de ambos respecto 
de la noción de individualidad. ${ }^{5}$ En segundo lugar, porque la mayoría de las interpretaciones sobre la relación entre Simondon y Marx suelen detenerse en las críticas de uno a otro sin reparar convenientemente en las afinidades que existen en cuestiones de otro orden que el trabajo y la alienación.

Concretamente, en La individuación... Simondon le reprocha a la concepción marxista la reducción de la realidad social, tanto en su aspecto vital como espiritual, al "[...] condicionamiento [...] de las superestructuras por la infraestructura económico-social.” (SIMONDON, 2009, p. 449). Para Simondon, la explotación de la naturaleza por el hombre, es decir, el trabajo, es un modo de conducirse en relación al medio, pero no es el único ni mucho menos aquél a partir del cual el hombre como tal puede ser definido. El trabajo sería una individuación a nivel físico y psíquico que permite la asociación y la disociación, esto es, la posibilidad de una vida que alterna la soledad con el agrupamiento, pero en ningún caso sería un rasgo que define al hombre en su infinita diversidad. El trabajo, entonces, puede considerarse una primera "individuación vital", pero luego de la cual el "ser humano se conserva aún inacabado, incompleto”. Ese algo que falta y que el trabajo por sí mismo no satisface totalmente es, según Simondon, una segunda individuación a la que denomina "individuación de grupo". Esta última, a diferencia de la primera, no se reduce a la relación del hombre con la naturaleza sino que exige una "base de espiritualidad en los grupos humanos". Aquello que Simondon entiende por espiritualidad no puede ser explicado sin alejarnos demasiado de nuestro problema, pero se puede precisar al menos que no es la simple oposición a la vida biológica ni depende del conocimiento metafísico o teológico. La espiritualidad - que desde esta perspectiva no queda confinada a los hombres sino que incluye como posibilidad a los animales — está ligada a la afectividad, a la sensación de la relación entre la existencia individual y su propio límite o su propio y necesario desborde.

La crítica a la noción de trabajo en Marx se condensa en estas pocas líneas del capítulo final de La individuación...:

Existen quizás otras infraestructuras que la explotación de la naturaleza por los hombres en sociedad, otros modos de relación con el medio que aquellos que pasan por la relación de elaboración, por el trabajo. La propia noción de infraestructura puede ser criticada: ¿el trabajo es una estructura, o bien una tensión, un potencial, una cierta forma de ligarse al mundo a través de una actividad que apela a una estructuración sin ser ella misma

\footnotetext{
${ }^{5}$ Sobre este punto, véase Balibar (2009).
} 
una estructura? Si se admite que los condicionamientos socionaturales al nivel específico son múltiples, es difícil extraer de allí uno y afirmar que posee valor de estructura; quizás Marx ha generalizado un hecho histórico real, a saber la dominancia de ese modo de relación con la naturaleza que es el trabajo en las relaciones humanas del siglo XIX; pero es difícil hallar el criterio que permita integrar esta relación a una antropología. (SIMONDON, 2009, p. 449).

La antropología implícita de Marx - implícita en tanto y en cuanto nunca se propuso desarrollar una ciencia positiva del hombre - fue y sigue siendo objeto de importantes debates dentro y fuera del marxismo. No hay consenso acerca de lo que Marx entiende por hombre justamente porque a lo largo de sus escritos - tanto en los que escribió solo como en aquellos que escribió con Engels — se observan aproximaciones muy diferentes a esta cuestión. No obstante, aquella que Simondon elige como blanco de su crítica es una de las más extendidas entre los marxistas occidentales: el hombre considerado como esencialmente trabajador o productor de valores. Más adelante habrá posibilidad de constatar que no es la única. Pero desde el punto de vista de Simondon, que es lo que ahora importa, no hay razón para semejante privilegio ya que el trabajo no sería para el hombre más que un modo entre otros de relación con el medio. ${ }^{6}$

La crítica esgrimida en El modo de existencia de los objetos técnicos es complementaria de la anterior. En este caso, está dirigida al concepto marxiano de alienación y se enmarca en una reevaluación general del problema de la técnica en el mundo contemporáneo. Simondon plantea aquí una exigencia que será constantemente relanzada a lo largo de su obra: “[...] suscitar una toma de conciencia del sentido de los objetos técnicos" (SIMONDON, 2008, p. 31), esto es, una toma de conciencia por parte de los hombres respecto de la relación que mantienen con las máquinas. Esta reconsideración es exigida tanto a nivel teórico como práctico. Por un lado se trata de reconocer la significación cultural de la realidad técnica más allá de su utilidad o función, y por otro lado y al mismo tiempo se trata de poner en práctica relaciones éticas más responsables y respetuosas para con los objetos técnicos. ${ }^{7}$

\footnotetext{
${ }^{6}$ La pregunta por el hombre y la antropología en Simondon es una enorme cuestión que no puedo tratar aquí como quisiera pero de la cual se hablará, directa o indirectamente, a lo largo de todo este trabajo. Para un examen en profundidad al respecto, véase Guchet (2010).

${ }^{7}$ Véase Bontems (2013).
} 
Uno de los objetivos principales de este libro es diferenciar el trabajo, entendido como actividad mediadora entre la especie y la naturaleza, de la operación técnica, del funcionamiento técnico y de la tecnicidad, para luego invertir la relación jerárquica entre estos términos. Simondon analiza críticamente el proceso histórico por el cual la técnica es reducida al trabajo, convirtiendo a este último en "[...] paradigma universal de la génesis de los seres.” (SIMONDON, 2008, p. 258). En este contexto, Simondon llega a afirmar que el trabajo por sí mismo es la fuente de la alienación:

[...] ni el marxismo, ni este contra-marxismo que es el psicologismo en el estudio del trabajo a través de las relaciones humanas, pueden encontrar la verdadera solución, porque ambos ubican la fuente de la alienación fuera del trabajo, mientras que el trabajo mismo en tanto que trabajo es la fuente de la alienación. (SIMONDON, 2008, p. 264).

Con esto no se trata de negar la existencia de la alienación económica, pero Simondon da a entender que ésta sería una alienación segunda respecto de una alienación primera o esencial que tiene su origen en el propio trabajo y que consiste en un total desconocimiento del objeto técnico. Por su parte, Marx entiende la alienación económica en dos sentidos: como alienación del trabajador respecto del producto de su trabajo y respecto del propio trabajo. En la medida en que el trabajador no es propietario de los medios de producción y está obligado a vender su fuerza de trabajo en el mercado, enfrenta el producto de su trabajo y el trabajo mismo como realidades ajenas, es decir, como realidades que no le son propias y que tampoco controla. El fenómeno de la alienación económica, así entendido, supone el modo de producción capitalista que le da origen. En consecuencia, la abolición de aquel depende de la abolición de este. Pero Simondon propone otra explicación, otra fuente y otro sentido de este fenómeno:

[la] alienación, cuya fuente el marxismo encuentra en la relación del trabajador con los medios de producción, no proviene solamente, en nuestra opinión, de una relación de propiedad o no propiedad entre el trabajador y los instrumentos de trabajo. Bajo esta relación jurídica y económica existe otra todavía más profunda y esencial, la de la continuidad entre el individuo humano y el individuo técnico, o de la discontinuidad entre ambos seres. [...] La alienación del hombre en relación con la máquina no tiene solamente un sentido económico-social; tiene también un sentido psico-fisiológico; la máquina ya no prolonga el esquema corporal, ni para los obreros ni para quienes las poseen. (SIMONDON, 2008, p. 135-136). 
En resumen, Simondon no crítica la noción marxiana de alienación por errónea, sino por insuficiente. Marx no habría visto más allá del aspecto jurídico y económico de la alienación; no habría visto la dimensión individual, psicológica y fisiológica de la alienación del hombre en relación con la máquina. La alienación de la que se habla aquí afecta por igual a proletarios y capitalistas en la medida en que unos y otros se encuentran en igual situación de incomprensión respecto de la técnica, ambos "[...] fallan en la relación verdadera con el objeto técnico individualizado bajo la forma de la máquina." Como han destacado diversos comentaristas, la originalidad del planteo de Simondon en este sentido corre en paralelo a una significativa desatención de su parte respecto de la dimensión social y política de la dominación en el análisis del trabajo y la alienación. ${ }^{8}$

Sea como fuere, no hay que perder de vista que más allá de las críticas mencionadas existe otra relación posible entre Simondon y Marx. Uno de los primeros intérpretes en llamar la atención sobre puntos de contacto entre las teorías de uno y otro fue el filósofo francés Étienne Balibar. Cuando apareció $\mathrm{La}$ filosofía de Marx (1993), libro en donde Balibar propone una lectura cruzada de Marx y Simondon, la obra de este último todavía era en buena medida desconocida. Como se ha dicho muchas veces, Gilles Deleuze fue durante décadas uno de los pocos autores contemporáneos a Simondon en advertir la importancia, riqueza y originalidad de sus conceptos. ${ }^{9}$ En ese contexto, la interpretación de Balibar inauguró una nueva perspectiva para pensar la relación entre Simondon y Marx y, de manera más general, la inscripción política del pensamiento simondoniano.

En la perspectiva abierta por Balibar cabe situar, asimismo, la lectura de Paolo Virno. En una serie de textos aparecidos a lo largo de la década del 2000, Virno hace dialogar a Simondon con Marx a fin de esclarecer y apuntalar su concepto de "multitud". Dicho diálogo y las consecuencias que de allí hace derivar aparecen desarrolladas principalmente en dos de sus libros más emblemáticos: Gramática de la multitud. Para un análisis de las formas de vida contemporáneas (2001) y Cuando el verbo se hace carne. Lenguaje y naturaleza humana (2003).

\footnotetext{
${ }^{8}$ Véase, por ejemplo, Combes (1999).

${ }^{9}$ Véase DELEUZE, 2005. Además de dedicarle esta temprana reseńa, Deleuze reafirma el valor de los conceptos de Simondon en algunos de sus libros más importantes, entre ellos, Diferencia y repetición (1968), Lógica del sentido (1969) y Mil mesetas (1980), este último escrito junto a F. Guattari.
} 
Al menos aquí, no es cuestión de realizar un estudio pormenorizado de estas lecturas. Las introduzco, entre otros motivos, porque son los dos antecedentes más importantes de un análisis de la relación entre Simondon y Marx que toma en consideración las afinidades entre sus respectivas propuestas. ${ }^{10}$ Aun cuando el alcance y los objetivos de estas interpretaciones son claramente diferentes, llama la atención que tanto Balibar como Virno hayan privilegiado la categoría de lo transindividual para dar cuenta de una intuición que, según ellos, Simondon y Marx habrían compartido.

Vale preguntarse, pues, ¿qué se juega en la noción de transindividual para que dos pensadores en apariencia tan alejados puedan ser acercados justamente a partir de ella?

\section{INDIVIDUALIDAD Y TRANSINDIVIDUALIDAD}

Para empezar conviene aclarar que el término "transindividual" es una invención bastante reciente. Hasta donde se sabe, fue introducido en 1952 por el filósofo francés Raymond Ruyer en un contexto espiritualista (BARTHÉLÉMY, 2008, p. 85-86), aunque ya aparece en las conferencias de Kojève sobre la Fenomenología del espiritu de Hegel dictadas entre 1933 y 1939 (KOJÈVE, 2013). Antes de Simondon, también fue utilizado por Lacan en su famoso "Discurso de Roma” (1953) para describir el inconsciente (LACAN, 2008). Ahora bien, el sentido que le otorga Simondon no se asemeja a ninguno de los anteriores y el lugar que ocupa al interior de su propia teoría tiene una centralidad que el vocablo no tiene para ninguno de los autores antes mencionados.

Se podría decir sencillamente que para Simondon lo transindividual es lo que ocurre en y a través de los individuos haciéndolos coincidir. Prácticamente no habría que agregar nada a esta definición si supiéramos de antemano qué es un individuo para Simondon. Pero he aquí todo el problema. En su teoría de la individuación, la cuestión de lo transindividual es inseparable del cuestionamiento a la noción de individuo tradicionalmente entendida, inseparable, pues, de la crítica al modo de entender el individuo como una realidad dada, constituida.

La dificultad más evidente para definir lo transindividual es que dicha categoría se encuentra en el corazón de un pensamiento abigarrado y complejo del que sería necesario dar cuenta en toda su amplitud, tarea que aquí, por si

${ }^{10}$ Aunque no son los únicos. Véanse también los trabajos de Read (2011) y Bardin (2013). 
hace falta aclararlo, es imposible llevar a cabo. No obstante, voy a intentar avanzar a partir de algunos indicios que permitan vislumbrar mínimamente lo que está en juego cuando se habla de transindividualidad.

A contrapelo de una larga tradición que se remonta hasta Aristóteles y llega hasta nosotros atravesando los más diversos saberes, Simondon parte de la individuación y no del individuo. Lo que se propone en La individuación... es "[...] conocer al individuo a través de la individuación antes que la individuación a partir del individuo." (SIMONDON, 2009, p. 26). Al invertir el punto de partida busca demostrar que el individuo, más que una realidad consumada hacia la cual el pensamiento tiende naturalmente, es una realidad parcial y relativa. El individuo, al que la abrumadora mayoría de las filosofías de todos los tiempos le confiere el estatuto de "modelo del ser", se presenta aquí como una fase del devenir del ser, precedido y sucedido por otras fases. El individuo, entonces, no es el ser absoluto sino que es devenir del ser. Devenir es la capacidad del ser para desfasarse respecto de sí mismo. Desde esta concepción, el ser tiene varias fases sucesivas, compatibles y relacionadas entre sí. El ser en tanto individuo es polifásico, lo que equivale a decir múltiple, plural, discontinuo...

De este modo, el privilegio ontológico del individuo es suspendido y desplazado hacia la individuación. Lo que importa ya no es el principio de individuación, sino la operación de individuación. Justamente, aquello en lo que coinciden las dos vías tradicionales de entender el individuo que Simondon identifica al comienzo de su libro, a saber, la vía sustancialista (que considera el individuo como una unidad existente por sí misma) y la hilemórfica (que considera el individuo como síntesis de materia y forma), es que "[...] ambas suponen que existe un principio de individuación anterior a la individuación misma, susceptible de explicarla, de producirla, de conducirla." (SIMONDON, 2009, p. 23). La operación de individuación es el movimiento por el cual el ser preindividual deviene ser individuado, es decir, individuo. Simondon llama ser preindividual a la fuente de toda individuación. El ser preindividual es definido como un sistema metaestable (concepto tomado de la termodinámica que significa, de manera aproximada, un estado de equilibrio poco estable que en determinadas circunstancias tiende a estabilizarse), un sistema - explica Simondon — rico en potenciales y fuerzas en tensión, esencialmente irresuelto y en situación de incompatibilidad consigo mismo. La "unidad" y la "identidad", principios característicos del ser individuado, no son aplicables al ser preindividual: "[...] esas nociones no pueden ayudar a descubrir el principio de individuación; no se aplican a la 
ontogénesis entendida en el sentido pleno del término, es decir al devenir del ser en tanto ser que se desdobla y se desfasa al individuarse." (SIMONDON, 2009, p. 27). El ser no tiene "unidad de identidad" sino "unidad transductiva", esto es, procesual, susceptible de transformación. La ontogénesis, o génesis del individuo, es otra forma de referirse a la individuación: operación sin principio a través de la cual es posible conocer el ser individuado.

A lo largo de su libro, Simondon describe tres niveles escalonados de individuación: el físico, el biológico y el psicosocial. A cada uno de ellos le dedica largos desarrollos, los cuales a su vez están ligados entre sí. Aquí voy a centrarme en la individuación psicosocial, pues es en el marco de esta reflexión que cobra toda su relevancia la cuestión de lo transindividual. No obstante, es importante tener en cuenta que si bien cada uno de estos niveles expresa una modalidad singular de individuación, la relación que guardan entre sí es de la mayor importancia para asegurar la coherencia general de la teoría de la individuación allí formulada.

En el último capítulo del libro, "Los fundamentos de lo transindividual y la individuación colectiva", Simondon distingue entre la individuación psíquica o interior, a la que dedica el penúltimo capítulo, y la individuación colectiva o exterior. La categoría de lo transindividual aparece allí, precisamente, para dar cuenta de la reciprocidad y de la "unidad sistemática" entre ambas individuaciones. El vínculo entre el individuo y lo transindividual es lo que "[...] supera al individuo mientras lo prolonga: lo transindividual no es exterior al individuo y sin embargo se aparta en cierta medida de él." (SIMONDON, 2009, p. 417). El carácter eminentemente ambiguo de esta definición tiene que ver con el hecho de que lo transindividual es considerado una suerte de límite, que une y separa a la vez, entre la interioridad y la exterioridad, entre lo psíquico y lo social, sin llegar a reconocerse plenamente en lo uno ni en lo otro. La realidad transindividual es psico-social: opera interiorizando lo exterior y exteriorizando lo interior simultánea y recíprocamente. En este sentido se puede afirmar que la acción transindividual atraviesa a los individuos, uno por uno y conjuntamente, dando lugar a la existencia conjunta. La individuación transindividual es cronológicamente posterior a la individuación biológica, aquella por la cual se constituye el ser viviente. La primera se superpone y desborda a la segunda. La individuación transindividual es la estructuración colectiva producida a partir de ese "fondo no estructurado" que subsiste como resto preindividual luego de la estructuración biológica. La individuación biológica, que tiene como resultado al individuo y al medio asociado a éste, no agota la totalidad de los potenciales y las fuerzas en tensión. Luego de esta primera individuación todavía hay resto 
en el individuo para una segunda individuación, ya no biológica sino colectiva o, mejor dicho, transindividual, en la que tiene un papel determinante la afectividad y la emotividad. A este resto preindividual que todo individuo lleva consigo Simondon lo llama naturaleza en el sentido presocrático de la palabra: naturaleza o ápeiron, literalmente, lo ilimitado, lo indefinido, lo indeterminado. La naturaleza, se lee al final de La individuación..., es "[...] realidad de lo posible". Tal vez, la frase que mejor capta el sentido profundo y a la vez simple de lo transindividual es aquella que el autor toma prestada a Malebranche y que aparece repetidas veces a lo largo de su libro: "tenemos movimiento para ir siempre más lejos"; "de hecho", reafirma Simondon, "tenemos tensión, potenciales para devenir otros, para recomenzar una individuación que no es destructora de la primera." (SIMONDON, 2009, p. 451).

Asimismo, Simondon pide no confundir lo transindividual con lo "social puro" (cuya existencia está garantizada por la individuación biológica de los individuos que conforman la sociedad, sin necesidad de una segunda individuación, y supone individuos heterogéneos tanto en lo estructural como en lo funcional) ni con lo "interindividual puro" (que tampoco necesita de una segunda individuación, y que supone analogía entre los individuos y reciprocidad en el intercambio). A diferencia de cualquier forma de vínculo social, lo transindividual es, ante todo y sobre todo, relación. ${ }^{11}$ En este punto es necesario comprender que para Simondon, contrariamente a lo que el discurso físico y metafísico afirma desde la antigüedad, la relación tiene "rango de ser". Esto significa que la relación no es un simple vínculo entre dos términos que tendrían una existencia independiente, sino que la relación es contemporánea de los términos que relaciona. Dicho de otro modo, la existencia de la relación es simultánea respecto de los términos relacionados. Desde el momento en que se abandona la concepción según la cual los términos son sustancias y la relación un accidente posterior y secundario respecto de los términos, se disloca la clásica jerarquía entre estas nociones, lo que hace posible pensar

\footnotetext{
${ }^{11}$ Simondon hace una distinción importante entre la palabra rapport, que en la versión castellana de La individuación... se traduce la mayoría de las veces por "vínculo", y la palabra relation, la cual se traduce por "relación": "[...] podemos llamar relación [relation] a la disposición de los elementos de un sistema que tiene una importancia que sobrepasa una simple visión arbitraria del espíritu, y reservar el término de vínculo [rapport] a una relación arbitraria, fortuita, no convertible en términos sustanciales; la relación sería un vínculo tan real e importante como los términos mismos; se podría decir en consecuencia que una verdadera relación entre dos términos equivale de hecho a un vínculo entre tres términos." (SIMONDON, 2009, p. 93). Más adelante, en el mismo libro, se argumenta que la relación posee "valor de realidad" (SIMONDON, 2009, p. 116), "valor de ser" (SIMONDON, 2009, p. 193), mientras que el vínculo tiene un carácter meramente formal, inesencial.
} 
la relación de un modo completamente diferente al habitual, a saber, "como relación en el ser, relación del ser, manera del ser”. La relación es individuación, es devenir, es ser que se desfasa respecto de sí mismo.

Este modo de concebir la relación es un postulado decisivo en la trama argumental de La individuación..., cuyos efectos se hacen sentir en el análisis de la individuación física, biológica y psicosocial. En lo que respecta a esta última, la única de la que aquí se trata, las consecuencias son inmensas. Quizás habría que empezar por decir que desde esta perspectiva, la oposición entre individuo y sociedad que atraviesa el discurso de las ciencias sociales desde sus orígenes pierde su pertinencia y, con ella, pierde también su eficacia. Ni el individuo ni la sociedad son realidades sustanciales que puedan existir independientemente la una de la otra y con anterioridad a la supuesta relación de oposición que se predica entre ellas. Hablar de existencia individual o de existencia social implica, pues, la relación necesaria entre una y otra. Tratar al individuo como anterior a la sociedad o bien a la sociedad como anterior al individuo es recaer, en el primer caso, en un sustancialismo de tipo psicológico, y en el segundo, en un sustancialismo de tipo sociológico. Tanto en un caso como en el otro lo que queda velado es la relación, la operación relacional, la actividad relacional que no es individual ni social sino la unidad desdoblada de ambas realidades y que a su vez permite una entrada diferente a las problemáticas respectivas del "individuo" y de la "sociedad". El psicologismo y el sociologismo, a los que Simondon considera "dos sustancialismos a diferentes niveles, moleculares o molares", desconocen por igual "la propia naturaleza de la relación social". Ahora bien, la crítica del enfrentamiento entre sociologismo y psicologismo no se resuelve en una tercera posición conciliadora, sea "microsociológica" o sea "macropsíquica", pues este tipo de soluciones siempre terminan por privilegiar uno de los términos de la oposición. La existencia psicosocial de la que habla Simondon refiere a una situación concreta que no parte de la "interioridad psíquica" ni de la "exterioridad social", sino de la relación intrínseca entre ambas realidades consideradas únicamente como casos límites. Entre una y otra encuentra su lugar y significación lo transindividual. La posición de Simondon respecto de la mentada oposición no deja lugar a dudas: "Lo social puro y lo individual puro existen por relación a la realidad transindividual, como términos extremos de toda la extensión de lo transindividual; lo individual y lo social no existen uno con relación al otro en calidad de términos antitéticos." (SIMONDON, 2009, p. 470). Esta frase, escrita justo antes de pasar a la "Conclusión", sintetiza una serie de fuertes cuestionamientos epistemológicos desarrollados en La individuación... que el 
autor lamentablemente no ampliará en trabajos posteriores sino de manera indirecta y muy general.

De lo dicho hasta aquí se deduce que lo transindividual no es individual ni social, o es ambas cosas a la vez. Detenta al mismo tiempo la fuerza oscilatoria del "entre" y la fuerza oblicua del "trans". En última instancia, lo que está en juego en esta apuesta por la transindividualidad es una nueva ontología o, como prefiere decir Simondon, una nueva ontogénesis. Vale decir, una ontología sin sustancia y ajena a cualquier tipo de sustancialismo, una ontología que, con toda razón, puede denominarse relacional. La tentativa simondoniana de fundar un nuevo pensamiento de la individuación tiene derivas teóricas y prácticas específicas en cada uno de los dominios donde opera. En lo que respecta a las humanidades y más particularmente a las ciencias sociales, aquella se encuentra estrechamente ligada a la posibilidad de llevar hasta sus últimas consecuencias la lógica paradójica de la transindividualidad.

\section{MarX y LA REALIDAD RELACIONAL}

En cierto modo, era necesario llegar hasta acá, hasta el punto preciso al que Simondon y algunos de sus lectores nos conducen en la actualidad, para reencontrar a Marx y a un conjunto de problemas que forman parte de su producción teórica. Entre otros, pero sobre todo, la crítica del modo fundamentalmente abstracto en que los pensadores de su tiempo (incluidos los más radicales) definían al individuo humano, el estudio del proceso de individualización del hombre en la vida colectiva, las advertencias sobre el riesgo y el error de promover la falsa oposición entre individuo y sociedad, etc. Hay que admitir de entrada que no son los problemas más resonantes de su legado. No obstante ello, se encuentran entre los más valiosos para un lector contemporáneo por ser aquellos que hoy, tanto por razones teóricas como por razones prácticas, es oportuno descubrir o redescubrir. En un momento en que Marx está en vías de convertirse en “un filósofo más” del que se puede hablar "serena y objetivamente, sin tomar partido: de acuerdo con las reglas académicas, en la universidad, en la biblioteca, en los coloquios", en un momento como éste sobre el que Derrida advertía premonitoriamente dos décadas atrás (DERRIDA, 2003, p. 45), sin duda es un buen síntoma el hecho de que la teoría marxiana sea revisitada para pensar asuntos actuales y no simplemente para rehacer una historia que en buena medida ya está acabada. 
No por azar los pasajes donde Marx evoca estos problemas han sido vinculados a la obra de Simondon. Como decía al comienzo, Balibar se encuentra entre los primeros intérpretes en haber detectado correspondencias entre ambos. Y lo hace, más precisamente, en una notable interpretación de las Tesis sobre Feuerbach (1845). Como se recordará, en las Tesis... Marx no solamente impugna la definición de Feuerbach sobre la esencia humana, sino que nos ofrece una definición completamente nueva de la misma. Dice el comienzo de la sexta tesis:

Feuerbach resuelve la esencia religiosa en la esencia humana. Pero la esencia humana no es algo abstracto e inmanente a cada individuo. Es, en su realidad, el conjunto de las relaciones sociales (Aber das menschliche Wesen ist kein dem einzelnen Individuum inwohnendes Abstraktum. In seiner Wirklichkeit ist es das ensemble der gesellschaftlichen Verhältnisse). (MARX, 1969; MARX, 1985, p. 667).

La radicalidad de la fórmula de Marx reside, según Balibar, en la recusación de las posiciones dominantes en torno a la cuestión de la naturaleza humana. Por una parte, Marx rechaza "a la vez las dos posiciones (llamadas realista y nominalista) entre las que se dividen tradicionalmente los filósofos: la que pretende que el género, o la esencia, precede a la existencia de los individuos, y la que quiere que los individuos sean la realidad primera, a partir de la cual se 'abstraen' los universales". Por otra parte, rechaza "a la vez el punto de vista individualista", que implica "primacía del individuo", y "el punto de vista organicista" u "holista", que implica "primacía del todo y en especial de la sociedad" (BALIBAR, 2006, p. 36-37). Al definir la esencia humana como el conjunto de las relaciones sociales, Marx desbarata de un solo golpe una serie de presupuestos fundamentales para la filosofía y las ciencias sociales. Aquellos sobre los que descansa la prevalencia de la sustancia (concebida como individualidad o como totalidad) sobre la relación o, mejor aún, sobre las relaciones. Las relaciones sociales que Marx hace coincidir con la esencia humana no son relaciones entre individuos constituidos ni tienen lugar en el seno de una sociedad ya existente. El conjunto de las relaciones sociales constituye tanto a los individuos como a la sociedad. De este modo, Balibar puede hablar de una "concepción de la relación constitutiva" en Marx (a este respecto, es notable, aunque Balibar no lo nota o al menos no lo hace notar, que la expresión "relación constitutiva” ya aparezca en La individuación... con un significado prácticamente idéntico al que tiene aquí). 
Así, pues, para caracterizar la concepción de la relación que se deduce de la sexta tesis sobre Feuerbach, Balibar propone utilizar la palabra transindividual: "[...] se trata de pensar la humanidad como una realidad transindividual y, en el límite, pensar la transindividualidad como tal.” (BALIBAR, 2006, p. $37-$ 38). Si bien advierte que el término fue empleado por diferentes pensadores del siglo XX, es sobre todo en el sentido de Simondon quo lo utiliza para leer a Marx. ${ }^{12}$ Por transindividual entiende: "No lo que está idealmente 'en' cada individuo (como una forma o una sustancia) o lo que serviría para clasificarlo desde el exterior, sino lo que existe entre los individuos, a raíz de sus múltiples interacciones." (BALIBAR, 2006, p. 38).

Balibar encuentra en el enunciado de Marx el esbozo de una "ontología de la relación", o también, de una "ontología de lo transindividual", que disloca la tradicional ontología de la sustancia. ${ }^{13}$ Tanto en el texto que venimos leyendo como en un texto del mismo autor bastante más reciente donde retoma y profundiza esta problemática (BALIBAR, 2012), se hace hincapié en la identificación que hace Marx entre el ser o la esencia (Wesen) del hombre en su realidad efectiva (Wirklichkeit) y la actividad o praxis. En la interpretación de Balibar, la frase "el conjunto de las relaciones sociales" (das ensemble der gesellschaftlichen Verhältnisse) habla de relaciones transformadoras y esencialmente heterogéneas con resonancias singulares en cada una de las esferas donde los individuos participan en su existencia común: política, trabajo, amor, etc. Esto quiere decir que no habría modelo o paradigma de la relación social, como tampoco habría un tipo de relación social más esencial y en consecuencia más determinante que otro. Justamente, lo único esencial aquí es el conjunto abierto y por lo tanto indeterminado, el ensemble (palabra francesa, no alemana, que a juicio de Balibar Marx habría intercalado deliberadamente para evitar el empleo de das Ganze, que refiere al "todo") de las relaciones sociales.

Paolo Virno es otro de los intérpretes en haber establecido puntos de encuentro entre Simondon y Marx. Incluso se puede decir que tiende a leerlos como autores compatibles y, hasta cierto punto, complementarios. Las categorías de uno y otro aparecen superpuestas, afirmándose mutuamente y afirmando a su vez el análisis de Virno sobre una serie de diferencias conceptuales fundamentales al interior de su propio trabajo: por un lado, la diferencia entre "pueblo" y "multitud", y por otro, la diferencia entre "reificación" y "fetichismo". ${ }^{14}$

\footnotetext{
${ }^{12}$ También se apoya sobre este concepto para leer a Spinoza. Véase, por ejemplo, Balibar (2009).

${ }^{13}$ Sobre esta cuestión, me permito remitir a mi artículo Alvaro (2012).

${ }^{14}$ Sobre la primera de estas diferencias, véase Virno (2003). Sobre la segunda, véase Virno (2005).
} 
No me detendré en ellas puesto que no es ese el objetivo. Aquí, simplemente, quiero llamar la atención sobre el concepto marxiano de "individuo social" que Virno examina en paralelo a la dimensión transindividual. La expresión "individuo social” es utilizada por Marx en el famoso "Fragmento sobre las máquinas" incluido en los Grundrisse (18571858). En el marco de un estudio sobre la relación entre capital y trabajo en la era de la gran industria, del desarrollo de la ciencia y del avance tecnológico, aparece el concepto de individuo social (gesellschaftliche Individuum), de cuyo desarrollo las fuerzas productivas y las relaciones sociales son aspectos diversos (MARX, 1983; MARX, 2011, p. 228-229). Virno (2005, p. 234) escribe:

A primera vista, el 'individuo social' parece un bello oxímoron, una confusa mezcla de contrarios, en suma un manierismo hegeliano. Pero es posible tomar este concepto al pie de la letra, a fin de hacer con él un instrumento de precisión para revelar modos de ser, inclinaciones y formas de vida contemporáneas. Pero esto es posible, en buena medida, gracias a las reflexiones de Simondon y de Vygotskij sobre el principio de individuación.

Por una parte, Virno nos exhorta a leer el concepto de individuo social de Marx en su radical literalidad y, consecuentemente, a reafirmar la coexistencia efectiva de lo individual y lo social en el animal humano con todo lo que ello conlleva desde un punto de vista ontológico y epistemológico. Por otra parte, aclara que si el concepto de Marx puede ser de utilidad para decir algo sobre el presente es gracias a los aportes ulteriores de Simondon y el psicólogo ruso L. S. Vygotskij, dos de los autores que más claramente privilegiaron el proceso de individuación en la búsqueda de una explicación a la ontogénesis.

Esta última aclaración es importante en la medida en que evita una posible confusión. Marx, por sí mismo, nunca desarrolló una ontología ni una epistemología en el sentido estricto de estos saberes. Su producción teórica, consagrada a la revolución social de la cual depende la realización individual, se inscribe en otros saberes. Por mucho que se busque entre sus textos, no se encontrará una reflexión formal y acabada sobre el principium individuationis y sus efectos sobre el conocimiento. Lo que hay son expresiones - con o sin estatuto conceptual - , frases breves y párrafos enteros, muchas veces de apariencia aporética o contradictoria, donde es posible leer el anuncio de un problema que no encuentra resolución inmediata y que por otra parte no siempre la busca. Con plena consciencia de esta situación, Virno ( 2005, p. 234) continúa: "En el adjetivo 'social' se debe reconocer la forma de aquella 
realidad preindividual que, según Simondon, pertenece a cada sujeto. Así como en el sustantivo 'individuo' se reconoce la singularización sucedida a cada uno de los componentes de la multitud actual" . La interpretación de la fórmula de Marx a la luz de categorías contemporáneas como la de realidad preindividual, singularidad o multitud, es la marca de una lectura que, como la de Balibar, intenta reactivar la herencia marxista transformando algunos de sus hallazgos teóricos en herramientas políticas para el presente.

Virno, entonces, interpreta el individuo social de Marx, "con la ayuda de Simondon", como una "[...] mezcla inextricable de singularidad contingente y realidad preindividual." (VIRNO, 2005, p. 236). Esta mezcla de singularidad y de pluralidad, de lo que es único y de lo que es común a todos, está en la base de la idea de transindividualidad. Sólo a partir de tal concepción del individuo y de la individuación se puede acceder a la realidad psicosocial o transindividual entendida como aquello que ocurre en y a través de los individuos.

Aún en la estela de esta reflexión podemos agregar que la expresión "individuo social" no debe ser confundida con una metáfora para indicar la naturaleza social del hombre como podría creerse con facilidad. Que el hombre es un animal social o un animal político ya lo sabía Aristóteles y no era necesario que Marx viniera a confirmarlo. Lo que Marx nos hace saber a través de este enunciado aparentemente contradictorio es que el individuo no sólo es inconcebible fuera de la sociedad, sino que el individuo, si se puede decir así, se individúa en sociedad, es decir, con otros individuos. En las primeras páginas de la "Introducción" (1857) que precede a los Grundrisse puede leerse esta famosa afirmación de Marx: "El hombre es, en el sentido más literal, un $\zeta \tilde{\omega} o v$

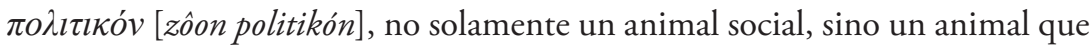
sólo puede individualizarse [sich vereinzeln, aislarse] en la sociedad." (MARX, 2007, p. 4). Ahora bien, decir que el individuo se individualiza —o se aíslaen la sociedad resulta problemático en la medida en que no hay individuo antes de la sociedad ni sociedad antes del individuo. Paradójicamente, eso es lo que Marx intenta explicar. Basta leer frases como ésta para comprender que buena parte de la dificultad que enfrentaron algunos de los teóricos más audaces que nos anteceden, y que nosotros, sus herederos, continuamos enfrentando, es que faltan categorías para nombrar una realidad relacional que no se ajusta en ningún sentido a la lógica clásica.

Sin duda, las citas de Marx podrían multiplicarse. De distintas formas, todos los pasajes de Marx que apuntan en esta dirección se apoyan directa o indirectamente en un postulado ontológico planteado tempranamente en 
los Manuscritos: "Hay que evitar, ante todo, que se vuelva a fijar la 'sociedad' ('Gesellschaft') como abstracción contrapuesta al individuo. Este último es el ser social (Das Individuum ist das gesellschaftliche Wesen)." (MARX, 1968; MARX, 2006, p. 145). El riesgo de contraponer la sociedad (palabra que Marx tiene la precaución de poner entre comillas) al individuo no es otro que comprender la realidad efectiva como una abstracción, y por lo tanto no comprenderla en absoluto. Sólo abstractamente se puede llegar a una comprensión del individuo y la sociedad como realidades diferentes. Fijarlas en abstracto, contraponiéndolas, es ignorar la movilidad o más exactamente el carácter procesual de la existencia. Decir, como hace Marx, que el individuo es el ser o la esencia social es un modo de afirmar, con una radicalidad verdaderamente inaudita para la época, la coexistencia originaria entre dos instancias que sólo analíticamente pueden existir en forma separada.

¿Cómo describir la lógica implicada en semejante postulado? ¿Cómo no ver aquí una prefiguración del planteo simondoniano sobre la transindividualidad? Afirmar que Marx es un pensador de lo transindividual avant la lettre no supone restarle ningún mérito a Simondon. La originalidad de su teoría de la individuación no se ve afectada por esta suerte de resonancia marxiana, remota y ciertamente intempestiva. Pero tampoco supone creer ingenuamente, anacrónicamente, que el razonamiento de uno es una simple anticipación o prolepsis del razonamiento del otro, como si éstos pudieran ser interpretados fuera del contexto histórico-conceptual en el que cada uno de ellos se inscribe. Dicha afirmación no tiene más pretensión que la de darle un lugar a Marx ahí donde Simondon no se lo da y donde aún hoy pocos se lo reconocen. El cuestionamiento de las posiciones sustancialistas y de las oposiciones binarias características del discurso científico-social o humanístico no depende de un autor particular ni de una disciplina específica. La deconstrucción de los conceptos fundamentales de ese discurso debe tomar en cuenta todos los legados disponibles que puedan favorecer esta tarea, sin prejuicios de origen o de filiación. Lo que realmente cuenta, más allá de la procedencia y de la identificación o de la inscripción disciplinaria, es la disposición de un pensamiento para la crítica tanto de los presupuestos teóricos como de los efectos políticos e ideológicos de una trama discursiva determinada. Aunque más no fuese por esta sola razón, en adelante habrá que contar con Marx, con Simondon, y con quienes antes o después de ellos, también imaginaron un nuevo punto de partida, una nueva lógica para las ciencias sociales o humanas. 
ALVARO, Daniel. The transindividual: from Simondon to Marx. Trans/form/ação, Marília, v. 39, n. 4, p.153-172, Out./Dez., 2016.

\begin{abstract}
The present article is about the transindividual, a fundamental concept in the theory of the individuation produced by Gilbert Simondon (1924-1989), and about its entailment with certain propositions of Karl Marx, that deal with the individual and the society. Between both authors there is a direct relationship, evidenced in each of the texts where Simondon makes mention to Marx and the Marxism in general. But it also exists an indirect relationship, at all evident, that arises less from what Simondon says about Marx than from what certain contemporary thinkers claim about one and the other. Here I attempt to give account of both types of relationships. In addition, I propose to introduce and comment some passages of the work of Marx, which compete in both radicalness and audacity with those that Simondon wrote a century after. And in which I want to sustain my reading hypothesis, namely, that Marx is a thinker of the transindividual avant la lettre.
\end{abstract}

KEY WORDS: Transindividual. Simondon. Marx. Relation.

\title{
REFERÊNCIAS
}

ALVARO, D. Marx y la ontología de lo común. Nómadas: Revista Crítica deCciencias Sociales y Jurídicas, v. 36, n. 2, p. 13-29, jul./dic. 2012.

BALIBAR, E. La filosofía de Marx. Buenos Aires: Nueva Visión, 1993.

. La filosofía de Marx. Traducción H. Pons. Buenos Aires: Nueva Visión, 2006.

. Spinoza: de la individualidad a la transindividualidad . Traducción A. Torres. Córdoba: Brujas, 2009.

From philosophical anthropology to social ontology and Back: what to do with Marx's sixth thesis on Feuerbach? Postmodern Culture,v. 22, n. 3, 2012 . Disponible en: <https://muse.jhu.edu/login?auth=0\&type=summary\&url=/journals/postmodern culture/v022/22.3.balibar.html> . Acceso en : 28 mar. 2015.

BARDIN, A. De l'homme à la matière: pour une "ontologie difficile" : Marx avec Simondon. Cahiers Simondon, n. 5, p. 25-43, 2013.

BARTHÉLÉMY, J.-H. Simondon ou l'encyclopédisme génétique. Paris: PUF, 2008.

BONTEMS, V. Esclaves et machines, même combat! L'aliénation selon Marx et Simondon. Cahiers Simondon, n. 5, p. 9-24, 2013.

COMBES, M. Simondon: individu et collectivité: pour une philosophie du transindividuel. Paris: PUF, 1999.

DE BOEVER, A. et. al. (Ed.). Gilbert Simondon : Being and technology. Edinburgh: Edinburgh University Press, 2012.

DELEUZE, G. Gilbert Simondon: el individuo y su génesis físico-biológica (1966): la isla desierta y otros textos: textos y entrevistas 1953-1974. Traducción J. L. Pardo. Valencia: Pre-textos, 2005. 
DERRIDA, J. Espectros de Marx. el estado de la deuda, el trabajo del duelo y la nueva internacional. Traducción. J. M. Alarcón y Cristina de Peretti. Madrid: Trotta, 2003.

GUCHET, X. Pour un humanisme technologique: culture, technique et société dans la philosophie de Gilbert Simondon. Paris: PUF, 2010.

KOJÈVE, A. Introducción a la lectura de Hegel. Traducción A. Alonso Martos. Madrid: Trotta, 2013.

LACAN, J. Función y campo de la palabra y del lenguaje en psicoanálisis. Escritos 1 . Traducción T. Segovia. Buenos Aires: Siglo XXI, 2008.

MARX, K. Ökonomisch-philosophische manuskripte aus dem Jahre 1844. Marx-Engels Werke Ergänzungsband 1. Berlin: Dietz, 1968.

. Thesen über Feuerbach. In: Marx-Engels Werke. Bd. 3. Berlin: Dietz, 1969.

. Grundrisse der kritik der politischen Ökonomie. In: Marx-Engels Werke. Bd. 42. Berlin: Dietz, 1983. S. 47-768.

- Tesis sobre Feuerbach. In: MARX, C.; ENGELS, F. La ideología alemana. Traducción W. Roces. Buenos Aires: Pueblos Unidos, 1985. p. 665-668.

. Manuscritos económico-filosóficos de 1844. Traducción M. Vedda, F. Aren y S. Rotemberg. Buenos Aires: Colihue, 2006.

. Elementos fundamentales para la crítica de la economía politica (Grundrisse) 18571858. Traducción P. Scaron. México: Siglo XXI, 2007. V. 1.

. Elementos fundamentales para la crítica de la economía política (Grundrisse) 18571858. Traducción P. Scaron. México: Siglo XXI, 2011. V.2.

READ, J. The production of subjectivity: from transindividuality to the commons. New Formations, n. 70, p. 113-131, Winter 2011.

SIMONDON, G. El modo de existencia de los objetos técnicos. Traducción M. Martínez y P. Rodríguez. Buenos Aires: Prometeo, 2008.

. La individuación a la luz de las nociones de forma y de información. Traducción P. Ires. Buenos Aires: Cactus; La Cebra, 2009.

VIRNO, P. Gramática de la multitud: para un análisis de las formas de vidas contemporáneas. Traducción A. Gómez, J. D. Estop, M. Santucho. Madrid: Traficantes de Sueños, 2003.

VIRNO, P. Cuando el verbo se hace carne: lenguaje y naturaleza humana. Traducción E. Sadier. Madrid: Traficantes de Sueños, 2005.

Recebido em 16/01/2016

Aceito em 19/05/2016 